J. Lake Sci. (湖泊科学), 2010, 22(1): 8-20

http: //www.jlakes.org. E-mail : jlakes@niglas.ac.cn

(c) 2010 by Journal of Lake Sciences

\title{
氮稳定同位素基准的可变性及对营养级评价的影响”
}

\author{
徐 军 ${ }^{1}$, 张 敏 ${ }^{2}$, 谢 平 ${ }^{1}$ \\ (1: 中国科学院水生生物研究所, 东湖湖泊生态系统试验站, 淡水生态与生物技术国家重点实验室, 武汉 430072) \\ (2: 华中农业大学水产学院,武汉 430070)
}

摘 要: 稳定同位素技术已经成为生态学家研究生态系统特征与过程的重要手段之一. 在利用稳定同位素研究植物一动 物和动物之间的营养关系或者进行多生态系统的比较研究过程中,通常需要选择某种生物的稳定同位素作为 “基准 (baseline)”. 同位素基准的目的是反映食物网生物最初物质来源的同位素特征. 同位素基准的选择取决于具体研究问题 与系统. 本文综述了水域生态系统研究中初级生产者和初级消费者氮稳定同位素作为基准的应用. 不同的研究都根据自 身所关注的生态学问题对基准进行选择和校正. 然而,找到适当的同位素基准取决于同位素基准种内、种间和时空上的 同位素变化特征,同时还取决于所关注的生态学问题的时间、空间尺度. 本文评估了季节性和种内的同位素变异性对消 费者营养级位置的影响.质量平衡模型的计算结果说明同位素基准可变性可以显著影响对消费者营养级位置的评估.

关键词: 水域生态学;稳定同位素;营养级;基准;初级生产者;初级消费者;指示生物;同位素分馏;周转速率

\section{Variability of stable nitrogen isotopic baselines and its consequence for trophic modeling}

\author{
XU Jun ${ }^{1}$, ZHANG $\operatorname{Min}^{2} \&$ XIE Ping ${ }^{1}$ \\ (1: Donghu Experimental Station of Lake Ecosystems, State Key Laboratory of Freshwater Ecology and Biotechnology of Chi- \\ na, Institute of Hydrobiology, Chinese Academy of Science, Wuhan 430072, P. R. China) \\ (2: College of Fishery, Huazhong Agricultural University, Wuhan 430070, P. R. China)
}

\begin{abstract}
Ecologists frequently use stable isotopes to measure the pattern and process of interested ecosystems. Comparisons of food web structure among and within ecosystems rely on a baseline isotopic signature for each system. The goal of a trophic baseline is to reflect the isotopic signature of the primary source of materials and nutrients for the food web. In general isotope baseline selection depends on the specific research question and must be tailored to a particular study system. We reviewed the use of the stable nitrogen isotopic signatures of primary producers and primary consumers to develop the baseline in aquatic ecology. Each of these studies used a slightly different method of baseline correction that was tailored to address a question of specific ecological interest. However, choosing an appropriate baseline depends on the inter- and intraspecific spatial, temporal variability of isotopic baselines and on the spatial and temporal context of the ecological question under consideration. We also evaluated to what extent isotopic heterogeneity actually influence consumer's trophic positions at the temporal and intraspecifical scales. The result of trophic position output estimated using an isotopic mixing model was generally variable, provided that baseline variability for trophic position estimation were sufficiently distinct in dietary mixing models.
\end{abstract}

Keywords: Aquatic ecology; stable isotope; trophic position; baseline; primary production; primary consumer; bioindicator; fractionation; turnover rate

稳定性同位素技术已经成为生态学家研究生态系统特征与过程,包括食物网结构、食物链长度、食物网 耦合等,探讨物质和能量在生物圈的流动和循环的重要手段之一 ${ }^{[1-8]}$. 它已经是研究生物之间的营养关系、 环境营养状况以及生物迁徙途径等问题的必要途径 ${ }^{[9-11]}$.生态系统食物网底层不同营养物质来源的稳定同 位素特征(包括内源与外源、沿岸带与敞水区、海洋与淡水、自然与人为等不同来源)都会影响食物网不同营

* 国家重点基础研究发展计划项目 (2008CB418001-1, 2007CB109205) 和国家自然科学基金项目 (30870428)联合资 助. 2009-04-10 收稿;2009-06-06 收修改稿. 徐军, 男,1978 年生, 博士, 副研究员; E-mail: xujun@ ihb. ac. cn. 
养级生物的稳定同位素组成 ${ }^{[12-16]}$, 同时随着物质在生态系统不同生物组分的循环过程会改变同位素组 成 ${ }^{[11]}$. 因此, 从生物个体到群落的稳定同位素特征均存在季节与空间的变化 ${ }^{[1,11,13]}$.

同位素生态研究过程中, 通常需要选择某种生物的稳定同位素作为 “基准” (Baseline), 用于评价生态系 统中高营养级生物功能或者进行多生态系统的比较研究. 基准生物 (物质) 通常为食物网中低营养级生物或 其有机物, 例如初级生产者或初级消费者或碎屑等. 基准生物的选择以及基准同位素的准确性对推断和分 析不同层次与尺度的生态学问题至关重要 ${ }^{[5,17-20]}$. 当评价研究对象历史变化过程的研究中, 如果基准本身不 能代表整个食物网物质基础, 或只能代表连续变化过程中 (季节变化或随机事件引起的变化) 的某一时间点 的特征, 那么就会对认识研究对象的特征与过程产生影响 ${ }^{[10,19]}$. 同时, 各种因素所引起的基准特征的改变也 会在食物网不同生物组分中以不同时间尺度体现出来, 这主要取决于生物的营养级位置、代谢特征、物质来 源等 ${ }^{[21-23]}$. 因此,许多学者建议对研究所需要的生物基准的同位素变化特征进行细致描述 ${ }^{[9-11,13,24]}$.

生态学家应用消费者的氮稳定同位素 $\left(\delta^{15} \mathrm{~N}\right)$ 平均值评价其营养级位置, 用种内或种间 $\delta^{15} \mathrm{~N}$ 的变化范围 评价其营养级宽幅. 评价生物营养级位置需要确定基准生物和它的 $\delta^{15} \mathrm{~N}$, 通过比较生物与基准的 $\delta^{15} \mathrm{~N}$ 及其 在营养级之间的富集常数可以计算出生物营养级位置 ${ }^{[5,13,25-26]}$. 在多生态系统比较研究中, 选择相同或类似 的 $\delta^{15} \mathrm{~N}$ 基准可以避免由营养级变化引起的误判 ${ }^{[13,17,27]}$. 但是由种群组成、食性转变、食物来源的时空变化等 因素所引起的基准生物种内和种群 $\delta^{15} \mathrm{~N}$ 的改变会影响到对高营养级生物在生态系统中的功能的评判 ${ }^{[28-30]}$. 同时, $\delta^{15} \mathrm{~N}$ 在食物链传递过程中富集特征也存在种内与种间变化, 因此认识某一基准物种 $\delta^{15} \mathrm{~N}$ 富集特征的 种内变化对解释种群 $\delta^{15} \mathrm{~N}$ 的变化有极大的帮助, 进而对基准生物的判别与选择具有指导意义 ${ }^{[13,21-22,31]}$.

生物食物来源的时空与组成的变化特征在某种意义上决定了基准生物的选择 ${ }^{[1,13,17,27,32-34]}$. 例如, 评价 鱼类营养来源对浮游与底栖食物网耦合的作用需要分别确定浮游与底栖食物网的基准生物, 包括蛙类、浮 游动物、螺类、摇蚊幼虫等,并假定这些初级消费者基准 $\delta^{15} \mathrm{~N}$ 在时间变化上是均一的 ${ }^{[5,13,35]}$. 因此,基准生物 的稳定性同位素季节变化在一定程度上可以通过加密时间尺度上样品密度以及选择同位素周转速度慢、寿 命长的生物来克服 ${ }^{[1,10,13,17]}$. 例如, Cabana 和 Rasmussen 选择 $>250 \mu \mathrm{m}$ 浮游动物作为评价鱼类营养级位置的 基准, 以消除初级生产者 $\delta^{15} \mathrm{~N}$ 随时间变化大所引起的误差 ${ }^{[1,32]}$. Vander Zanden 和 Vadeboncoeur 等利用蚌 类、螺类、摇蚊幼虫等生活史周期相对较长的初级消费者的 $\delta^{15} \mathrm{~N}$ 评价鱼类在食物网耦合中的作用及其杂食

性 ${ }^{[35-36]}$. Post 通过比较初级消费者与初级生产者的 $\delta^{15} \mathrm{~N}$ 发现, 蛙类、螺类 $\delta^{15} \mathrm{~N}$ 可以代表初级生产者 $\delta^{15} \mathrm{~N}$ 季 节变化的平均特征, 因此可以用作浮游与底栖食物网的基准. Matthews 和 Mazumder 指出在研究浮游食物网 结构和功能的过程中植食性浮游动物 Daphnia 的 $\delta^{15} \mathrm{~N}$ 可以用作同位素基准, 并提出了基于此基准的浮游食 性鱼类营养级特征季节变化的评价模型 ${ }^{[27,33,37]}$.

综上所述, 同位素基准变化通过食物链传递作用最终会影响到生态系统食物网不同营养级各生物类群 的同位素特征, 进而会影响生态学家对食物网结构与功能的评判. 因此, 就所关注的生态学问题如何选择适 合的生物基准, 以及如何准确把握基准 $\delta^{15} \mathrm{~N}$ 的变化特征, 对生态学家有效利用稳定同位素技术至关重要. 本 文简单介绍氮稳定同位素及其在水域生态学中的应用, 并着重综述了此类研究中各类基准生物的 $\delta^{15} \mathrm{~N}$ 主要 变化特征, 并且分析了基准 $\delta^{15} \mathrm{~N}$ 变化对生态系统结构与功能评价的影响, 以期对进一步深人水域生态系统 的同位素生态学研究起到一定促进作用.

\section{1 氮稳定同位素及其水域生态学中的应用}

\section{1 氮稳定同位素特征}

自然界中氮在大气中的含量中 $99.637 \%$ 是 ${ }^{14} \mathrm{~N}$, 而剩余的 $0.363 \%$ 是 ${ }^{15} \mathrm{~N}$. 实际测定氮稳定性同位素的过 程中, 通常采用相对测量法测定, 即将所测样品的氮同位素比值与相应的标准物质的同位素比值作比较, 比 较结果称为样品的氮稳定性同位素比率, 即 $\delta^{15} \mathrm{~N}$ 值. 其公式为:

$$
\delta^{15} \mathrm{~N}(\%)=\left(\frac{\left({ }^{15} \mathrm{~N} /{ }^{14} \mathrm{~N}\right)_{\text {sample }}}{\left({ }^{15} \mathrm{~N} /{ }^{14} \mathrm{~N}\right)_{\text {standard }}}-1\right) \times 10^{3}
$$

式中, 当 $\delta^{15} \mathrm{~N}$ 为正值时, 表明样品的重同位素含量高于标准物质的含量; 当 $\delta^{15} \mathrm{~N}$ 为负值时, 表明样品的重同 
位素含量低于标准物质的含量. 大气中 ${ }^{15} \mathrm{~N}$ 与 ${ }^{14} \mathrm{~N}$ 的比值就是氮稳定性同位素测量中的国际标准 ${ }^{[38-39]}$, 其 $\delta^{15} \mathrm{~N}$ 值被定义为 $0 \%$ 。

\section{2 氮稳定同位素的分馏}

在生态系统能量传递的过程中, 氮同位素存在同位素分馏作用, 这导致了生物体与其能量来源的稳定 性同位素比率的差异. 此过程中的同位素分馏为消费者与食物之间的稳定性同位素变化,主要包括对不同 同位素组成的食物的选择性吸收和同位素辨别两个过程 ${ }^{[40]}$. 与食物相比, 消费者的氮稳定性同位素比率约 增加 3\% $5 \%$. 其原因为消费者的排泄物中尿素和氨的氮稳定性同位素相对较低, 从而导致了高营养级生物 的氮稳定性同位素相对较高 ${ }^{[21,41]}$. 生物稳定性同位素的富集同时受到食性、代谢机能、环境、饵料的质量以 及样品采集和处理等一系列因素的影响 ${ }^{[21-22]}$. 例如, 动物细胞内谷氨酸和天冬氨酸之间的转氨作用过程 中, ${ }^{14} \mathrm{NH}_{2}$ 比 ${ }^{15} \mathrm{NH}_{2}$ 快 1.0083 倍, 这导致了食物缺乏的情况下动物组织 ${ }^{15} \mathrm{~N}$ 的增加 ${ }^{[42]}$.

\section{3 氮稳定同位素的应用}

氮稳定同位素在示踪不同污染来源的研究中有很强的优势 ${ }^{[2,43]}$. 溶解态硝酸盐的氮稳定同位素可以区 分不同来源的含氮污染物, 包括农业面源、养殖污染物和城市污水等 ${ }^{[4-45]}$. 此外, 废水处理过程中导致氨挥 发的工艺(例如高温、碱性条件)也会引起氮同位素的分馏,进而导致轻同位素随挥发带走,而保留下来的氮 库重同位素明显富集 ${ }^{[46]}$. 不同氮形态的商业肥料 $\delta^{15} \mathrm{~N}$ 值几乎相同 ${ }^{[47]}$, 且使用后在厌氧下通过反硝化作用 和氨的挥发等自然作用, $\delta^{15} \mathrm{~N}$ 值逐渐升高, 但显著低于人类或动物有机废物的 ${ }^{15} \mathrm{~N}^{14} \mathrm{~N}$ 值 $(>10 \%){ }^{[48]}$. 动物 产生的有机废物 $\delta^{15} \mathrm{~N}$ 在 $10 \%-20 \%$ 之间, 因此可以应用 $\delta^{15} \mathrm{~N}$ 区分动物性有机废物和工业合成化肥等氮 源 ${ }^{[4-45,49]}$, 但无法区分人类和动物间有机污染物氮源 ${ }^{[49]}$. 尽管不同研究区域同一氮源 $\delta^{15} \mathrm{~N}$ 存在一定差异, 但是同一区域内主要类型氮源 $\delta^{15} \mathrm{~N}$ 之间通常是有显著差异的. 近年来, 通过生物的氮稳定同位素来指示人 类活动引起环境污染的研究也越来越多 ${ }^{[32,34,50-51]}$, 包括植物、沉积物、无脊椎动物、鱼类等 ${ }^{[50-54]}$. 蚌类的代谢 速率远低于藻类、水生高等植物和小型的底栖动物, 且食物来源较鱼类单一, 因此在评价长期污染输人环境 效应中有较好的准确性 ${ }^{[1,51,55]}$. 氮通过大气与地表径流进人水体被初级生产者和微生物利用后通过食物网 进人动物组织, 通过排泄和分解作用重新返回水体 ${ }^{[56]}$. 营养物质在生态系统非生物与生物组分之间循环过 程中, 营养物与基准生物的稳定氮同位素特征存在着直接或间接的联系, 并且不同组分指示环境同位素变 化的程度与时效存在差异性与互补性. 基于消费者与其食物间稳定性同位素比率的关系, 即氮稳定性同位 素比率约增加 3\% 0 - 5\%, 氮稳定性同位素比率已经广泛地用于水域生态学食物网与氮循环的研究中 ${ }^{[4,56-58]}$. 例如 Peterson 等人利用附着藻类吸收铵的过程中产生的同位素分馏追踪氮在河流食物网中传递特征, 证明 了 Kuparuk 河流中消费者能量主要来自藻类, 而非陆源有机物 ${ }^{[3]}$.

\section{4 消费者营养级位置的估算}

$\delta^{15} \mathrm{~N}$ 也可以用来分析食物的贡献比例, 由于 $\delta^{15} \mathrm{~N}$ 的富集较大 (通常为 $3 \%-4 \%$ ), 所以 $\delta^{15} \mathrm{~N}$ 多用于消费 者营养级的评价, 其公式如下:

$$
\text { 营养级 }=\lambda+\frac{\delta^{15} N_{\text {消费者 }}-\delta^{15} N_{\text {基准 }}}{\Delta \delta^{15} \mathrm{~N}}
$$

式中, $\delta^{15} \mathrm{~N}_{\text {基准 }}$ 为食物网中初级生产者或初级消费者的 $\delta^{15} \mathrm{~N}$, 即 $\lambda=1$ 时, $\delta^{15} \mathrm{~N}_{\text {基准 }}$ 为初级生产者 $\delta^{15} \mathrm{~N}$; 而 $\lambda=2$

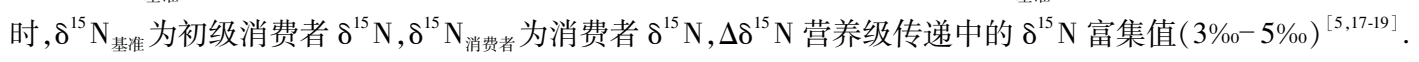

当消费者利用两种 $\delta^{15} \mathrm{~N}$ 不同的食物来源时, 以一种食物 $\delta^{15} \mathrm{~N}$ 作为同位素基值显然是不合理的. 因此,

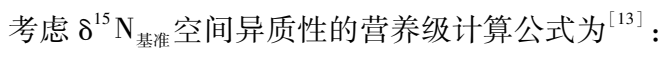

$$
\text { 营养级 }=\lambda+\frac{\delta^{15} \mathrm{~N}_{\text {消费者 }}-\left[\left(\delta^{15} \mathrm{~N}_{\text {基准 }} \alpha+\delta^{15} \mathrm{~N}_{\text {基准 } 2}(1-\alpha)\right)\right]}{\Delta \delta^{15} \mathrm{~N}}
$$

式中, $\alpha$ 为两种食物中的一种对消费者氮的贡献比例. 假定碳和氮元素沿食物链传递过程相似, 则 $\alpha$ 的计算 公式为:

$$
\alpha=\frac{\delta^{13} \mathrm{C}_{\text {消费者 }}-\delta^{13} \mathrm{C}_{\text {基准 }}}{\delta^{13} \mathrm{C}_{\text {基准 } 1}-\delta^{13} \mathrm{C}_{\text {基准 } 2}}
$$


该混合模型适用条件包括两种食物来源同位素存在差异、 $\delta^{13} \mathrm{C}$ 富集接近或者等于 $0 \%$ 以及两种同位素 混合过程为线性 ${ }^{[13]}$. 在一个食物网中最大的营养级也被定义为最大食物链长度或食物网高度 ${ }^{[5,17-19]}$.

综上所述, 氮同位素已经广泛用于污染物示踪及其生物累积效应、评价生物不同营养来源和营养关系、以 及氮的生物地球化学循环等生态学核心问题的研究中 ${ }^{[59]}$. 而选择适当的同位素基准是定量评价与准确分析生 态系统结构与功能的基础, 也就是说选择何种初级生产者或初级消费者的同位素比率来应用于模型之中 ${ }^{[60]}$. 由于藻类、浮游动物、小型底栖动物的个体较小, 受环境变化影响较大, 所以其同位素比率变化也较大, 在定量 评价营养关系的过程中会带来许多问题 ${ }^{[5,13,26,50,60-63]}$. 因此, 有些研究建议使用食性来源单一、长寿命的初级消 费者,例如多年生滤食性的蚌和底栖刮食性的螺等,作为代表不同食物网的同位素基准 ${ }^{[5,13,17,26,49]}$.

\section{2 氮稳定同位素基准及其变化特征}

\section{1 藻类与悬浮颗粒有机物}

藻类与悬浮颗粒有机物的 $\delta^{15} \mathrm{~N}$ 作为基准多用于浮游食物网营养关系、能量流动特征以及生物地球化学 循环的分析 ${ }^{[62,64-67]}$. 澡类的种类组成、生长特征、密度以及它所利用的溶解无机氮源的同位素特征均会影响 藻类 $\delta^{15} \mathrm{~N}$ 的变化 ${ }^{[63,66,68-70]}$. 此外, 影响藻类生长的因素, 例如光、水温、营养盐浓度, 和水体中氮循环的主要 过程, 例如硝化作用、反硝化作用、固氮作用等, 也会影响藻类 $\delta^{15} \mathrm{~N}$ 组成 ${ }^{[63,66,71]}$. 通常在湖水溶解无机氮充足 或浮游植物生长缓慢的条件下, 溶解无机氮和浮游植物的 $\delta^{15} \mathrm{~N}$ 相差最大, 而在溶解无机氮缺乏或浮游植物 生长迅速的条件下, 溶解无机氮和浮游植物的 $\delta^{15} \mathrm{~N}$ 相差最小 ${ }^{[30,70,72]}$. 固氮藻类利用了大气中的氮气 $\left(\delta^{15} \mathrm{~N}\right.$ 为 $0 \% 0$ ) 作为其氮源, 导致其 $\delta^{15} \mathrm{~N}$ 接近 $0 \%{ }^{[70,72-73]}$. Vuorio ${ }^{[68]}$ 分离了自然水体中的多种藻类并分析了 $\delta^{15} \mathrm{~N}$, 发 现藻类种间 $\delta^{15} \mathrm{~N}$ 差异很大, 其中固氮藻类 Anabaena spp. 和 Aphanizomenon spp. $\delta^{15} \mathrm{~N}$ 范围为 $0.5 \%-2.0 \%$. 反 硝化过程中, 氮同位素存在明显的分馏, 即轻同位素随氮挥发, 而剩余的氮库重同位素含量升高; 非戻氧水 体中, 硝化作用将轻同位素含量高的氨态氮转换成为亚硝酸盐和硝酸盐 ${ }^{[63]}$. 因此, 浮游植物利用不同的氮营养 盐形态也会引起其 $\delta^{15} \mathrm{~N}$ 的变化 ${ }^{[63,66,74]}$. 底栖附着藻类不仅是水域生态系统底栖或沿岸带食物网重要营养基 础, 近期研究还发现它也是浮游动物的食物来源, 对浮游食物网结构与功能的有一定的影响 ${ }^{[75]}$. 底栖藻类的 $\delta^{15} \mathrm{~N}$ 的变化除了受到以上因素的影响外还受到水流速度、固着厚度及其特有的代谢途径等 ${ }^{[76-78]}$. 除了浮游植 物, 颗粒有机物通常还包括其它微小生物和有机碎屑 ${ }^{[79]}$, 因此水体不同来源的有机颗粒物也会影响其 $\delta^{15} \mathrm{~N}$. 例 如, 内源与外源颗粒有机物 $\delta^{15} \mathrm{~N}$ 有明显差异, 湖泊地理位置、形态、流域植被类型以及人类活动污染物排放等 均会影响颗粒有机物 $\delta^{15} \mathrm{~N}^{[2,80-82]}$. 湖泊营养水平和生产力是影响悬浮有机颗粒物 $\delta^{15} \mathrm{~N}$ 的重要因素 ${ }^{\left[{ }^{[} 0,62,66,79,83\right]}$.

尽管许多研究报道了颗粒有机物 $\delta^{15} \mathrm{~N}$ 具有明显季节和空间变化 ${ }^{[50,63,65-66,70]}$, 但是仍然有许多研究仅依赖 少量的藻类或颗粒有机物样品的 $\delta^{15} \mathrm{~N}$ 作为基准评价营养关系与能流特征. 为了更全面准确地理解生态系统的 结构与功能, 建议通过长时间序列、高采样频度的样品采集, 找出 $\delta{ }^{15} \mathrm{~N}$ 的变化特征. 特别是在中、富营养水体中, 藻 类或颗粒有机物样品的 $\delta^{15} \mathrm{~N}$ 的季节变化极其显著, 通常可达 $5 \%-25 \%{ }^{[66,70,72,79]}$. 在 $\delta^{15} \mathrm{~N}$ 随食物网富集指示为 $3.4 \%$ 的情况下 ${ }^{[13,21-22]}$, 如此大的变化所引起营养关系误判是无法通过一次或短期的样品分析所能弥补的.

\section{2 浮游动物}

早期研究多使用浮游动物 $\delta^{15} \mathrm{~N}$ 作为湖泊浮游食物网的基准 ${ }^{[13,17,32-33]}$, 且通常使用整体或某一大小范围 的浮游动物, 不考虑种类组成可能引起的同位素变化. 实际上, 浮游动物不同种类组成的稳定同位素差异显

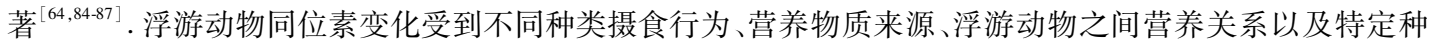
类同位素基准值的影响 ${ }^{[64,84,86-90]}$, 且不同浮游动物种类存在明显的同位素季节变化 ${ }^{[33,86,91-92]}$. 例如, 不同种类 浮游动物可以选择性的摄食不同种类或不同质量的藻类, 且同位素的富集特征也会发生变化, 进而影响浮 游动物的同位素组成 ${ }^{[61,86]}$. 因此,整体或某一大小范围的浮游动物其同位素高度异质性可能会影响系统内 或系统之间的比较研究, 这部分取决于所采集的某一大小范围浮游动物的组成在多大程度上反映了鱼类的摄 食情况. 例如在安大略湖, Kiriluk 等发现在 Bosmina 和硅藻类大量存在的情况下, 网采浮游生物 ( >0.153mm) $\delta^{15} \mathrm{~N}$ 为 $1.2 \%$, 而在剑水蚤占主体的情况下为 $12.1 \%{ }^{[93]}$. 用整体或某一大小范围的浮游动物 $\delta^{15} \mathrm{~N}$ 作为基准估 算鱼类的营养级也有其局限性. Matthews 和 Mazumder 通过综述已有浮游动物的同位素研究发现, 浮游动物 $\delta^{15} \mathrm{~N}$ 湖内某采样点变异系数最大, 其可能的原因为初级生产者同位素变化大以及浮游动物不同大小组成的变 
化 ${ }^{[33]}$. 如果浮游动物群的同位素差异产生源自营养来源的差异, 而非其内部营养关系变化, 那么整体或某一大 小范围的浮游动物的同位素特征就可能准确代表浮游动物群落的同位素变动的平均值 ${ }^{[33]}$.

另一种方式就是选择已知摄食习性的单个种类浮游动物 $\delta^{15} \mathrm{~N}$ 作为基准 ${ }^{[13,64]}$. 例如 Kling 等用已知为植 食性的桡脚类浮游动物作为评价其它杂食性桡足类浮游动物的基准. 其优点在于基准与其评价的生物有相 似的生物学特征, 并且同位素时空变化、代谢周转等特征也极为相似 ${ }^{[64]}$. Matthews 和 Mazumder 认为浮游动 物 Daphnia 为专一植食性, 且处于浮游食物链营养级为 2 , 因此应用浮游动物 Daphnia $\delta^{15} \mathrm{~N}$ 季节变化的平均 值作为评价鱼类营养级关系的基准 ${ }^{[27]}$. 此外, 利用 Daphnia $\delta^{15} \mathrm{~N}$ 季节平均值作为基准, 评价不同颗粒大小或 种类的浮游动物之间的营养级关系 ${ }^{[27]}$. 由于同位素基准需要依据所研究的内容和系统进行选择, 因此对浮 游动物进行分种分析同位素特征并不一定适用于所有问题 ${ }^{[13,27,37]}$.

浮游动物的 $\delta^{15} \mathrm{~N}$ 季节性变化非常突出, 且可能与营养级变动的关系不大. 消费者 $\delta^{15} \mathrm{~N}$ 代表了食物网中 氮循环在消费者所在营养水平的整体状况, 加之浮游植物 $\delta^{15} \mathrm{~N}$ 变动的不确定性, 浮游动物 $\delta^{15} \mathrm{~N}$ 的季节性变 动并非一定与营养级变动有关 ${ }^{[33,56,66]}$. O'Reilly 等在坦噶尼喀湖发现伴随 ${ }^{15} \mathrm{NH}_{4}$ 的上涌, 浮游动物 $\delta^{15} \mathrm{~N}$ 值快 速上升, 与浮游食性的消费者 $\delta^{15} \mathrm{~N}$ 相近, 证明了浮游动物 $\delta^{15} \mathrm{~N}$ 值可以因为氮循环的迅速改变而变动 ${ }^{[10]}$. 尽 管如此, 浮游动物 $\delta^{15} \mathrm{~N}$ 显著的季节性变化说明使用季节性平均值或是浮游动物时间序列 $\delta^{15} \mathrm{~N}$ 值评价浮游 食物网高营养级生物 (例如浮游食性鱼类) 营养位置的结果将会不同 ${ }^{[33]}$.

\section{3 底栖无脊椎动物}

摇蚁幼虫是大多数湖泊生态系统的重要组成部分, 其营养来源在很大程度上依赖于沉积的浮游植物和有 机碎屑等 ${ }^{[94]}$. 鉴于此, 在不能直接获得浮游生物稳定同位素的情况下, 摇蚊幼虫的同位素特征也被用于代表湖 泊浮游生物时间变化的综合指示基准同位素值的完整估计 ${ }^{[5,35-36]}$. 此类研究多依赖于有限的季节变化样品, 并 普遍认为摇蚊同位素变化特征远小于待评价的食物网不同组分的同位素变化关系. 然而,氮矿化作用、摇蚊新 陈代谢的季节性变化以及由于气温、氧气、食物供给的改变而导致的生长发育均会引起不同生活史阶段摇蚊 稳定性同位素特征的变化. 因此, 在使用摇蚊作为食物网基准生物过程中, 认识摇蚊幼虫同位素变化特征,对 示踪源含氮有机物生物地球化学循环特征、评价高营养级营养关系是十分必要的 ${ }^{[95-96]}$. 摇蚊幼虫成蛹羽化后, 剩余幼虫种群的平均稳定同位素组成也会发生变化. 因此, 不同种群和不同湖泊摇蚊生活史模式差异将会引 起剩余幼虫种群稳定同位素的季节性变化模式的差异 ${ }^{[96]}$. 此外, 摇蚊幼虫同位素特征存在种间差异 ${ }^{[07]}$. Grey 等对 Chironomus anthracinus 和 C. plumosus 进了同位素比较湖沼学研究, 发现不同湖泊摇蚊的 $\delta^{15} \mathrm{~N}$ 种内变化特 征差异很大, 且厌氧生境的种间变化最大. 其中两个湖泊 C. plumosus 的 $\delta^{15} \mathrm{~N}$ 种内变化达到 $16 \%$, 相当于跨越 了 5 个营养级, 反映出巨大的生态位宽幅以及独特的摄食行为 ${ }^{[95]}$. 也有研究报道了 $\delta^{15} \mathrm{~N}$ 指示的底栖动物生态 位重叠以及空间变化, 其原因可能是无机氮源及其可利用性的差异导致的 ${ }^{[98]}$. Goering 等报道了北太平洋一个 海湾的生物同位素季节变化, 发现浮游植物、浮游动物和 Macoma nasuta 的季节变化明显, 但是小型底栖动物 (大于 $98 \%$ 为线虫类) 同位素无显著季节变化 ${ }^{[99]}$. 一些土壤无脊椎动物的研究也证实了种内与种间 $\delta^{15} \mathrm{~N}$ 显著 差异的存在, 以及不同器官的同位素周转速率的差异 ${ }^{[100-101]}$. Lancaster 等通过分析溪流中底栖无脊椎动物的种 群 $\delta^{15} \mathrm{~N}$ 变化特征, 发现肉食性底栖动物 Rondania dorsalis $\delta^{15} \mathrm{~N}$ 种群内差异可达 $11 \%$, 且受到不同的种群同位素 差异受到重复抽样样品数量的影响 ${ }^{[102]}$. Zah 等还发现大型底栖动物的同位素特征受到内外源营养物比例、食物质 量及其同位素变化、摄食行为与可塑性及其较低的 $\delta^{15} \mathrm{~N}$ 营养级富集特征等一系列因素的影响 $(2.25 \%)^{[103]}$.

蚌类和螺类也广泛用作系统内不同区域和不同系统之间敞水区与沿岸带食物网特征比较的同位素基 准生物. Post 证明了蚌类和螺类的稳定同位素含量可以综合地反映敞水区与沿岸带食物网底层的同位素特 征 ${ }^{[13]}$. 蚌类除了用作研究淡水生态群落营养关系的基准生物, 也可以作为营养物污染负荷和来源的指示生 物 ${ }^{[13,34,52-53,104-106]}$. 淡水蚌类同位素组成也具有组织间、种内、种间和时空变化, 但相对于水体中营养物质或初 级生产者的同位素变化要小的多 ${ }^{[107-109]}$. 蚌类作为同位素基准广泛使用的主要原因是它活动范围小、生命周 期相对长、易采集、分布广泛、营养级位置低 (初级消费者) 且综合反映初级生产者的同位素特征. 蛙类主要 是通过滤食水体中的藻类、有机碎屑和微生物作为其主要食物来源 ${ }^{[1,13,34,107,110]}$. 不同生活史阶段蚌类摄食方 式的变化会影响其同位素特征. Goering 等在 Auke 海湾发现成年 Macoma nasuta 同位素表现出与浮游动植 物相反的季节变化特征 ${ }^{[99]}$. Fry 证明 Potamocorbula amurensis 的 $\delta^{15} \mathrm{~N}$ 可以指示人类活动导致的流域含氮污 
染物的输人以及流域人类活动对次级生产力变化的长期生态影响 ${ }^{[05]}$. Rossi 等通过分析不同个体大小的 M. balthica 和 C. edule 的同位素变化, 发现了 M. balthica 个体发育过程中食性由底栖微藻向浮游微藻的转 变, 且两种蚌类的营养级存在部分重叠 ${ }^{[111]}$. Wen 等也证实了富营养湖泊蚌类 $\delta^{15} \mathrm{~N}$ 存在种内、种间以及时空 等变化, 且与环境营养物变化之间有一定关系 ${ }^{[109]}$. 蚌类不同器官同位素富集特征也有差 ${ }^{\left[巳{ }^{[112]}\right.}$. Mckinney 研 究发现珠蚌的内收肌 $\delta^{15} \mathrm{~N}$ 比腹足和肌膜高 $1 \%$, 且在用单个蚌 $\delta^{15} \mathrm{~N}$ 作为基准对营养级评估过程中对一个营 养级最大可产生 0.56 的影响 ${ }^{[113]}$. Gustafson 等报道了蚌类 Elliptio complanata 的 $\delta^{15} \mathrm{~N}$ 与其生境中 $\mathrm{NO}_{3}$ 的 $\delta^{15} \mathrm{~N}$ 有显著关系, 并通过实验证明了蛙类血淋巴 $\delta^{15} \mathrm{~N}$ (周转周期 $113 \mathrm{~d}$ ) 对环境 $\mathrm{NO}_{3}$ 的 $\delta^{15} \mathrm{~N}$ 变化的缓慢响应 ${ }^{[107]}$.

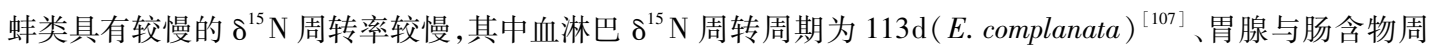
转周期分别为 $78 \mathrm{~d}$ 与 $85 \mathrm{~d}$ 、肌膜组织为 $120-180 \mathrm{~d}$ (Pinctada fucata martensii) ${ }^{[114]}$ 、肌肉组织为 $357 \mathrm{~d}$ (Pleurobe$m a$ sp. $)^{[115]}$ 、个体总体周转周期 $333 \mathrm{~d}$ (Mytilus edulis $)^{[116]}$, 因此, 其低周转率更适合用于判断环境中长期的生 态学与环境化学变动的指示 ${ }^{[107]}$. 螺类主要以藻类和有机碎屑为食 ${ }^{[117]}$. 螺类 $\delta^{15} \mathrm{~N}$ 的变化的研究相对较少, Wen 和 $\mathrm{Xu}$ (未发表) 的研究结果表明富营养湖泊螺类 $\delta^{15} \mathrm{~N}$ 有明显的季节变化, 且与水体中悬浮颗粒物 $\delta^{15} \mathrm{~N}$ 呈正相关; 此外, 螺类 $\delta^{15} \mathrm{~N}$ 也存在一定的种内与种间差异, 但是较同一栖息地的蚌类要小. 因此,尽管蚌类和 螺类 $\delta^{15} \mathrm{~N}$ 的变化较初级生产者或者同位素周转较快的初级消费者要小, 但是充足的种群、空间和季节性样 本对系统内或系统间问题的解释仍然重要.

\section{3 氮稳定同位素基准变异对营养级估算的影响评价}

选择适当的基准是有效使用同位素技术评价生态系统结构与功能的基础. 而通常使用的基准生物同位 素特征多存在种内、种间以及时空变化. 在营养级估算过程中, 这些变化对高营养级生物的评价直接影响到 生态学家对系统结构与功能的评判 ${ }^{[118]}$. 那么基准的变化对营养级评价究竟有多大的影响, 以及如何影响数 据的解释? 下面, 我们通过模拟基准的季节变化和种间差异, 通过质量平衡模型估算营养级变化, 分析其对 结果分析的潜在影响.

营养级是能量到达某一消费者能量所传递的级数, 因此营养级之间的关系是指一类生物和处于不同营养 层次上另一类生物之间的关系 ${ }^{[119-120]}$. 在同位素生态学研究中, 除了初级生产者与初级消费者, 其它消费者营 养级位置为非整数, 反映了生物之间营养关系的连续性 ${ }^{[17,58]}$. 依据营养级评价的同位素质量平衡模型 (公式 2), 对基准季节变化与种间变化对消费者营养级变化的影响进行评估. 在此评价过程中营养级富集因子为 $3.4 \%{ }^{[13,41,58]}$; 消费者同位素种内变化极小、周转周期极长, $\delta^{15} \mathrm{~N}=11.9 \% 0-\infty \%(n=+\infty)$, 且食物链消费者 与食物为简单捕食关系. 通过随机产生采样频次和样本数模拟季节变化 [基准同位素 $=$ 变幅 $\times \sin (x)$; 采样时 间 $\left.=\left(x /\left(x_{\max }-x_{\min }\right)\right) \times 365\right]$ 与种内变化 (正态分布随机函数) 的基准同位素特征对消费者营养级进行计算. 所有数据来源均为数学模拟. 总体来讲, 季节变化对营养级的大小和宽幅均有影响, 而种内变化仅对营养级宽 幅产生影响 (图 1 和图 2). 就季节变化而言, 本模拟中基准 $\delta^{15} \mathrm{~N}$ 变化范围 - $5 \% 0$ 至 5\% 之间, 季节性密集采样 $(N=120)$ 与常规采样 $(N=12)$ 所获得基准 $\delta^{15} \mathrm{~N}$ 在评价消费者营养级过程反映出的营养特征一致, 但营养级宽 幅极大 (3-6) ; 而局部时间段的基准 $\delta^{15} \mathrm{~N}$ 评价的营养级宽幅较小 $(<1)$, 但却高估或低估了消费者营养级. 就 种内变化而言, 尽管本模拟中种间变化的标准偏差从 $0.2-1.4$ 之间变动, 但对消费者营养级平均特征影响不 大, 而对营养级宽幅影响很大 (最大变幅为 3-6). 根据公式 2 , 影响消费者营养级评价的因素还包括富集因子 和消费者 $\delta^{15} \mathrm{~N}$ 的变化, 本评估过程尚未涉及. 但是, 已有研究表明氮稳定同位素富集因子在不同物种和不同生 态系统中存在变化 ${ }^{[13,21-22,121-122]}$. 生物稳定性同位素富集同时受到食性、代谢机能、环境、饵料的质量以及样品采 集和处理等一系列因素的影响 ${ }^{[13,21-22,121-122]}$. 此外, 当所评价的消费者食物源自两种或两种以上且不同基准的 $\delta^{15} \mathrm{~N}$ 有显著差异时, 评价营养级的同位素质量平衡模型需要考虑除 $\delta^{15} \mathrm{~N}$ 之外的其他同位素 (例如公式 2). 因 为在这种情形下, 即使消费者 $\delta^{15} \mathrm{~N}$ 组成相近或相似, 其所处的营养级位置也会有显著差异 ${ }^{[13,18,60]}$.

\section{4 结论与展望}

在过去的二十多年间, 应用生态学家发现了稳定性同位素在食物网研究中的潜力, 但是该方法的成功 应用还有赖于同位素基准生物的正确选择. 在同位素生态学研究中, 某种生物是否可以充当适当的同位素 

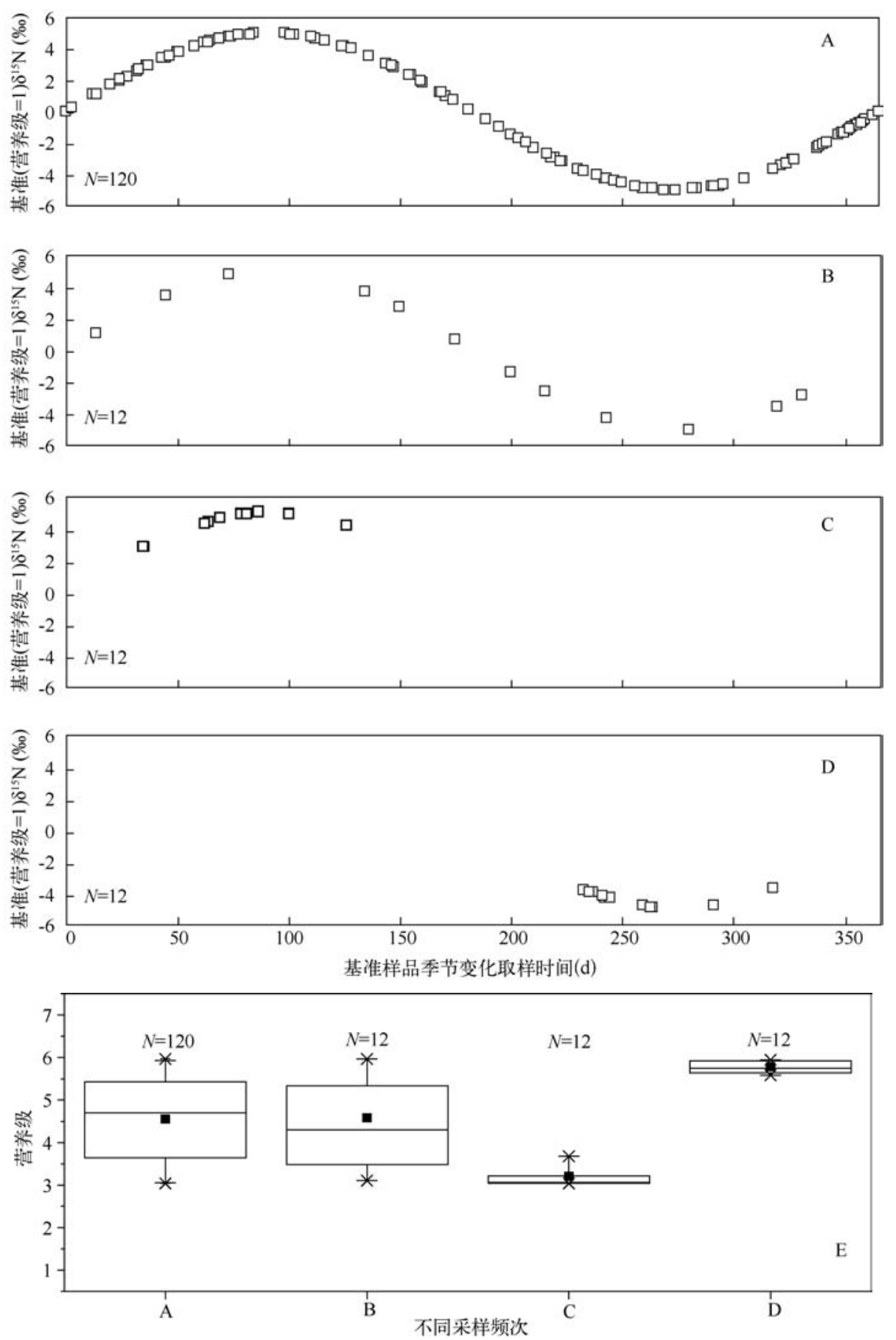

图 1 基准同位素季节变化对营养级评价的影响 $(A 、 B 、 C 、 D$ 为不同采样时间与采样频度的 基准稳定同位素特征; $\mathrm{E}$ 为四种采样方式所获得同位素基准的营养级评价结果; $N$ 为采样频次）

Fig. 1 Effect of seasonal variability in isotopic baselines on the calculation of trophic position

基准取决于它以何种方式、在何种时间尺度上对环境物质的吸收利用. 食性转变实验证明了消费者同位素 周转周期要大于其食物的同位素周转, 因此消费者反映食物同位素特征通常是滞后的 ${ }^{[123-124]}$, 因此高周转率 

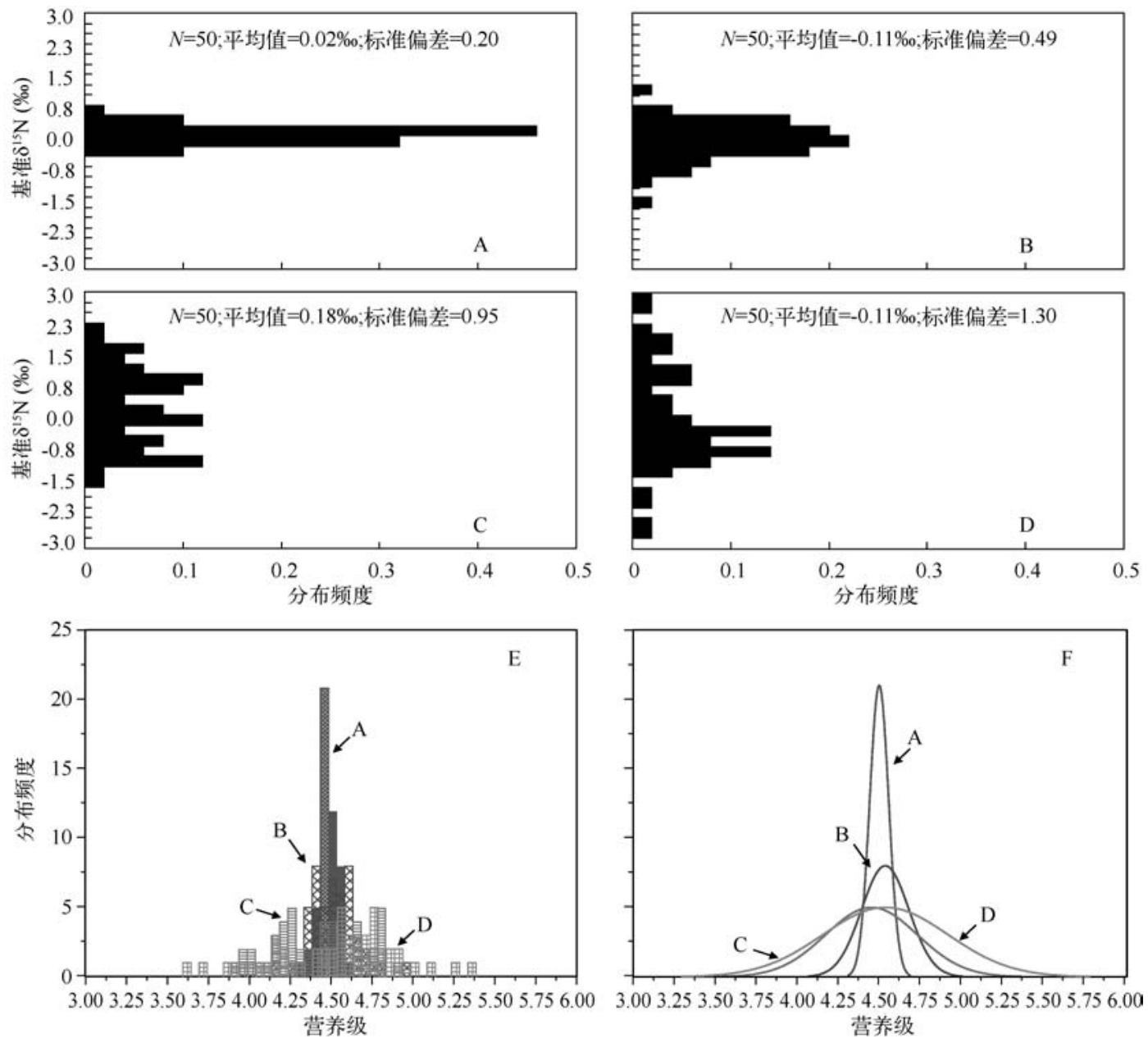

图 2 基准同位素种内变化对营养级评价的影响 ( A、B、C、D 为不同种内变化分布特征;

$\mathrm{E} 、 \mathrm{~F}$ 为不同种内分布特征的同位素基准营养级评价结果分布频度与正态分布特征）

Fig. 2 Effect of intraspecies variability in isotopic baselines on the calculation of trophic position

可以反映环境短期内的变动, 而低周转率则可以判断环境中长期的变动. 选择同位素基准的前提是根据所 研究的生态学问题,弄清潜在生物基准稳定同位素组成的组织间、种内、种间、时空等变化特征, 同时弄清其 在营养关系评价中的有效性, 以便提供较为精确的评价. 高周转率生物基准, 例如藻类或浮游动物, 短期或 某一时间点的样品采集分析不能提供同位素基准的完整特征, 通常需要分析其季节性的变化特征. 低周转 率生物基准对环境变化缓慢响应, 可以用作环境中营养关系水平中长期的综合指示生物, 可以作为相对稳 定和完整的同位素基准. 由于不同类型的同位素基准的变化发生在不同时间尺度上,且营养富集、同位素富 集和未知营养传递代谢途径均存在变化, 因此高周转率生物基准季节性的连续分析与低周转率生物基准长 期监测可以对分析系统短、中、长期的结构与功能变化提供有选择性的、互补优势的解释.

致谢: 本文中部分资料的收集与整理得益于华中农业大学水产学院杨陈与湖北大学资源环境学院吴丽英的 帮助, 在此一并致谢!

\section{5 参考文献}

[ 1 ] Cabana G, Rasmussen JB. Comparison of aquatic food chains using nitrogen isotopes. Proceedings of the National Academy 
of Sciences of the United States of America, 1996, 93: 10844-10847.

[ 2 ] Mcclelland JW, Valiela I. Linking nitrogen in estuarine producers to land-derived sources. Limnology and Oceanography, $1998, \mathbf{4 3}(4)$ : 577-585.

[ 3 ] Peterson BJ. Stable isotopes as tracers of organic matter input and transfer in benthic food webs: a review. Acta Oecologica, 1999, 20 : 479-487.

[ 4 ] Peterson BJ, Fry B. Stable isotopes in ecosystem studies. Annual Review of Ecology and Systematics, 1987, 18: 293-320.

[ 5 ] Vander Zanden MJ, Fetzer WW. Global patterns of aquatic food chain length. Oikos, 2007, 116: 1378-1388.

[ 6 ] Layman CA, Arrington DA, Montan ACG et al. Can stable isotope ratios provide for community-wide measures of trophic structure? Ecology, 2007, 88: 42-48.

[ 7 ] Thompson RM, Hemberg M, Starzomski BM et al. Trophic levels and trophic tangles: the prevalence of omnivory in real food webs. Ecology, 2007, 88: 612-617.

[ 8 ] Wada E, Kabaya Y, Kurihara Y. Stable isotopic structure of aquatic ecosystems. Journal of Biosciences, 1993, 18: 483-499.

[ 9 ] Gannes LZ, O'Brien DM, Del Rio CM. Stable isotopes in animal Ecology: assumptions, caveats, and a call for more laboratory experiments. Ecology, 1997, 78: 1271-1276.

[10] O'Reilly CM, Hecky RE, Cohen AS. Interpreting stable isotopes in food webs: recognizing the role of time averaging at different trophic levels. Limnology and Oceanography, 2002, 47: 306-309.

[11] Rubenstein DR, Hobson KA. From birds to butterflies: animal movement patterns and stable isotopes. Trends in Ecology and Evolution, 2004, 19: 256-263.

[12] France RL. Differentiation between littoral and pelagic food webs in lakes using stable carbon isotopes. Limnology and Oceanography, 1995, 440: 1310-1313.

[13] Post DM. Using stable isotopes to estimate trophic position: models, methods, and assumptions. Ecology, 2002,83 : 703 718 .

[14] France R. Stable nitrogen isotopes in fish: literature synthesis on the influence of ecotonal coupling. Estuarine, Coastal and Shelf Science, 1995, 41 : 737-742.

[15] Showers WJ, Williams CM, Jennings GD. Impact of large poultry operations on groundwater: stable ${ }^{15} \mathrm{~N}$ isotopes of nitrate assessment. International Journal of Poultry Science, 2006, 5 : 318-329.

[16] Wipfli MS, Hudson JP, Caouette JP et al. Marine subsidies in freshwater ecosystems: salmon carcasses increase the growth rates of stream-resident salmonids. Transactions of the American Fisheries Society, 2003, 132 : 371-381.

[17] Vander Zanden MJ, Casselman JM, Rasmussen JB. Stable isotope evidence for the food web consequences of species invasions in lakes. Nature, 1999, 401: 464-467.

[18 ] Vander Zanden MJ, Shuter BJ, Lester N et al. Patterns of food chain length in lakes: a stable isotope study. The American Naturalist, 1999, 154: 406-416.

[19] Post DM, Pace ML, Hairston Jr NG. Ecosystem size determines food-chain length in lakes. Nature, 2000, 405: 1047-1049.

[20] Sweeting CJ, Jennings S, Polunin N. Variance in isotopic signatures as a descriptor of tissue turnover and degree of omnivory. Functional Ecology, 2005, 19: 777-784.

[21] Mccutchan Jr JH, Lewis Jr WM, Kendall C et al. Variation in trophic shift for stable isotope ratios of carbon, nitrogen, and sulfur. Oikos, 2003, 102: 378-390.

[22] Caut S, Angulo E, Courchamp F. Variation in discrimination factors $\left(\delta^{15} \mathrm{~N}\right.$ and $\left.\delta^{13} \mathrm{C}\right)$ : the effect of diet isotopic values and applications for diet reconstruction. Journal of Applied Ecology, 2009, 46: 443-453.

[23] Polis GA, Anderson WB, Holt RD. Toward an integration of landscape and food web Ecology: the dynamics of spatially subsidized food webs. Annual Review of Ecology and Systematics, 1997, 28 : 289-316.

[24] Barnes C, Jennings S, Polunin N et al. The importance of quantifying inherent variability when interpreting stable isotope field data. Oecologia, 2008, 155: 227-235.

[25] Xu J, Xie P. Studies on the food web structure of Lake Donghu using stable carbon and nitrogen isotope ratios. Journal of Freshwater Ecology, 2004, 19: 645-650.

[26] Xu J, Zhang M, Xie P. Size-related shifts in reliance on benthic and pelagic food webs by lake anchovy. Ecoscience, $2007,14: 170-177$. 
[27] Matthews B, Mazumder A. Compositional and interlake variability of zooplankton affect baseline stable isotope signatures. Limnology and Oceanography, 2003, 48: 1977-1987.

[28 ] Maruyama A, Yamada Y, Rusuwa B et al. Change in stable nitrogen isotope ratio in the muscle tissue of a migratory goby, Rhinogobius sp., in a natural setting. Canadian Journal of Fisheries and Aquatic Sciences, 2001, 58: 2125-2128.

[29] Melville AJ, Connolly RM. Spatial analysis of stable isotope data to determine primary sources of nutrition for fish. Oecologia, 2003,136 : 499-507.

[30 ] Needoba JA, Waser NA, Harrison PJ et al. Nitrogen isotope fractionation in 12 species of marine phytoplankton during growth on nitrate. Marine Ecology Progress Series, 2003, 255 : 81-91.

[31] Post DM. Individual variation in the timing of ontogenetic niche shifts in largemouth bass. Ecology, 2003, 84: 1298-1310.

[32] Cabana G, Rasmussen JB. Modelling food chain structure and contaminant bioaccumulation using stable nitrogen isotopes. Nature, 1994, 372 : 255-257.

[33 ] Matthews B, Mazumder A. Consequences of large temporal variability of zooplankton $\delta^{15} \mathrm{~N}$ for modeling fish trophic position and variation. Limnology and Oceanography, 2005, 50: 1404-1414.

[34] Vander Zanden MJ, Vadeboncoeur Y, Diebel MW et al. Primary consumer stable nitrogen isotopes as indicators of nutrient source. Environmental Science and Technology, 2005, 39: 7509-7515.

[35] Vadeboncoeur Y, Jeppesen E, Schierup HH et al. From Greenland to green lakes: cultural eutrophication and the loss of benthic pathways in lakes. Limnology and Oceanography, 2003, 48: 1408-1418.

[36] Vander Zanden MJ, Chandra S, Allen BC et al. Historical food web structure and restoration of native aquatic communities in the Lake Tahoe (California-Nevada) basin. Ecosystems, 2003, 6: 274-288.

[37] Matthews B, Mazumder A. Detecting trophic-level variation in consumer assemblages. Freshwater Biology, $2008, \mathbf{5 3}$ : 1942-1953.

[38 ] Mariotti A. Atmospheric nitrogen is a reliable standard for natural ${ }^{15} \mathrm{~N}$ abundance measurements. Nature, 1983,303 : 685 687.

[39] Mariotti A. Natural ${ }^{15} \mathrm{~N}$ abundance measurements and atmospheric nitrogen standard calibration. Nature, 1984, 311: 251 252.

[40 ] Hobson KA, Clark RG. Assessing avian diets using stable isotopes I: turnover of ${ }^{13} \mathrm{C}$ in tissues. Condor, 1992, 94: 181-188.

[41] Minagawa M, Wada E. Stepwise enrichment of ${ }^{15} \mathrm{~N}$ along food chains: further evidence and the relation between ${ }^{15} \mathrm{~N}$ and animal age. Geochimica et Cosmochimica Acta, 1984, 48 : 1135-1140.

[42] Macko SA, Estep M, Engel MH et al. Kinetic fractionation of stable nitrogen isotopes during amino acid transamination. Geochimica et Cosmochimica Acta, 1986, 50 : 2143-2146.

[43] Chang C, Kendall C, Silva SR et al. Nitrate stable isotopes: tools for determining nitrate sources among different land uses in the Mississippi River Basin. Canadian Journal of Fisheries and Aquatic Sciences, 2002, 59: 1874-1885.

[44] Karr JD, Showers WJ, Jennings GD. Low-level nitrate export from confined dairy farming detected in North Carolina streams using $\delta^{15} \mathrm{~N}$. Agriculture, Ecosystems and Environment, 2003, 95: 103-110.

[45] Karr JD, Showers WJ, Hinson TH. Nitrate source identification using $\delta^{15} \mathrm{~N}$ in a ground water plume near an intensive swine operation. Ground Water Monitoring and Remediation, 2002, 22 : 68-75.

[46] Heaton THE. Isotopic studies of nitrogen pollution in the hydrosphere and atmosphere: A review. Chemical Geology, 1986, 59: 87-102.

[47] Gormly JR, Spalding RF. Sources and concentrations of nitrate-nitrogen in ground water of the central platte region, Nebraska. Ground Water, 1979, 17: 291-301.

[48 ] Flipse WJ, Bonner FT. Nitrogen-isotope ratios of nitrate in ground water under fertilized fields, Long Island, New York. Ground Water, 1985, 23 : 59-67.

[49] Curt MD, Aguado P, Sanchez G et al. Nitrogen isotope ratios of synthetic and organic sources of nitrate water contamination in Spain. Water, Air and Soil Pollution, 2004, 151: 135-142.

[50] Xu J, Xie P, Zhang M et al. Icefish (Salangidae) as an indicator of anthropogenic pollution in freshwater systems using nitrogen isotope analysis. Bulletin of Environmental Contamination and Toxicology, 2007, 79: 323-326.

[51] Cole ML, Valiela I, Kroeger KD et al. Assessment of a $\delta^{15} \mathrm{~N}$ isotopic method to indicate anthropogenic eutrophication in aquatic ecosystems. Journal of Environmental Quality, 2004, 33 : 124-132. 
[52] Lake JL, Mckinney RA, Osterman FA et al. Stable nitrogen isotopes as indicators of anthropogenic activities in small freshwater systems. Canadian Journal of Fisheries and Aquatic Sciences, 2001, 58: 870-878.

[53] Mckinney RA, Lake JL, Charpentier MA et al. Using mussel isotope ratios to assess anthropogenic nitrogen inputs to freshwater ecosystems. Environmental Monitoring and Assessment, 2002, 74: 167-192.

[54] Schlacher TA, Liddell B, Gaston TF et al. Fish track wastewater pollution to estuaries. Oecologia, 2005, 144: $570-584$.

[55] Kaushal SS, Lewis Jr WM, Mccutchan Jr JH. Land use change and nitrogen enrichment of a Rocky Mountain watershed. Ecological Applications, 2006, 16: 299-312.

[56] Robinson D. $\delta^{15} \mathrm{~N}$ as an integrator of the nitrogen cycle. Trends in Ecology and Evolution, 2001, 16: 153-162.

[57] Hansson S, Hobbie JE, Elmgren R et al. The stable nitrogen isotope ratio as a marker of food-web interactions and fish migration. Ecology, 1997, 78 : 2249-2257.

[58 ] Vander Zanden MJ, Rasmussen JB. A trophic position model of pelagic food webs: impact on contaminant bioaccumulation in lake trout. Ecological Monographs, 1996, 66: 451-477.

[59] Hobson KA. Tracing origins and migration of wildlife using stable isotopes: a review. Oecologia, 1999, 120: 314-326.

[60] Vander Zanden MJ, Rasmussen JB. Primary consumer $\delta^{13} \mathrm{C}$ and $\delta^{15} \mathrm{~N}$ and the trophic position of aquatic consumers. Ecolo$g y, 1999, \mathbf{8 0}: 1395-1404$

[61 ] Aberle N, Malzahn AM. Interspecific and nutrient-dependent variations in stable isotope fractionation: experimental studies simulating pelagic multitrophic systems. Oecologia, 2007, 154: 291-303.

[62 ] Gu B, Chapman AD, Schelske CL. Factors controlling seasonal variations in stable isotope composition of particulate organic matter in a softwater eutrophic lake. Limnology and Oceanography, 2006, 51 : 2837-2848.

[63] Hadas O, Altabet MA, Agnihotri R. Seasonally varying nitrogen isotope biogeochemistry of particulate organic matter in Lake Kinneret, Israel. Limnology and Oceanography, 2009, 54: 75-85.

[64] Kling GW, Fry B, O’Brien WJ. Stable isotopes and planktonic trophic structure in arctic lakes. Ecology, 1992, 73 : 561566.

[65] Gu B, Schell DM, Alexander V. Stable carbon and nitrogen isotopic analysis of the plankton food web in a subarctic lake. Canadian Journal of Fisheries and Aquatic Sciences, 1994, 51 : 1338-1344.

[66] Gu B. Variations and controls of nitrogen stable isotopes in particulate organic matter of lakes. Oecologia, 2009, 160: 421-431.

[67 ] Zhang L, Xu J, Xie P et al. Stable Isotope Variations in Particulate Organic Matter and a Planktivorous Fish in the Yangtze River. Journal of Freshwater Ecology, 2007, 22: 383-386.

[68 ] Vuorio K, Meili M, Sarvala J. Taxon-specific variation in the stable isotopic signatures $\left(\delta^{13} \mathrm{C}\right.$ and $\left.\delta^{15} \mathrm{~N}\right)$ of lake phytoplankton. Freshwater Biology, 2006, 51: 807-822.

[69] Xu J, Xie P, Qin J. Diel isotopic fluctuation in surface seston and its physiological and ecological implications. Annales de Limnologie-International Journal of Limnology, 2008, 44: 197-201.

[70] Syvaranta J, Tiirola M, Jones RI. Seasonality in lake pelagic $\delta^{15} \mathrm{~N}$ values: patterns, possible explanations, and implications for food web baselines. Fundamental and Applied Limnology, 2008, 172 : 255-262.

[71] Owens NJP. Natural variations in ${ }^{15} \mathrm{~N}$ in the marine environment. Advances in Marine Biology, 1987, 24 : 389-451.

[72] Yoshioka T, Wada E, Hayashi H. A stable isotope study on seasonal food web dynamics in a eutrophic lake. Ecology, 1994,75 : 835-846.

[73] Kendall C, Silva SR, Kelly VJ. Carbon and nitrogen isotopic compositions of particulate organic matter in four large river systems across the United States. Hydrological Processes, 2001, 15: 1301-1346.

[74] Lehmann MF, Bernasconi SM, Mckenzie JA et al. Seasonal variation of the $\delta^{13} \mathrm{C}$ and $\delta^{15} \mathrm{~N}$ of particulate and dissolved carbon and nitrogen in Lake Lugano: constraints on biogeochemical cycling in a eutrophic lake. Limnology and Oceanography, 2004, 49: 415-429.

[75] Karlsson J, Sawstrom C. Benthic algae support zooplankton growth during winter in a clear-water lake. Oikos, 2009,118 : 539-544.

[76] Macleod NA, Barton DR. Effects of light intensity, water velocity, and species composition on carbon and nitrogen stable isotope ratios in periphyton. Canadian Journal of Fisheries and Aquatic Sciences, 1998, 55 : 1919-1925.

[77 ] Trudeau V, Rasmussen JB. The effect of water velocity on stable carbon and nitrogen isotope signatures of periphyton. Limnology and Oceanography, 2003, 48 : 2194-2199. 
[ 78 ] Jones RI, King L, Dent MM et al. Nitrogen stable isotope ratios in surface sediments, epilithon and macrophytes from upland lakes with differing nutrient status. Freshwater Biology, 2004, 49 : 382-391.

[ 79 ] Xu J, Xie P, Zhang M et al. Variation in stable isotope signatures of seston and a zooplanktivorous fish in a eutrophic Chinese lake. Hydrobiologia, 2005,541 : 215-220.

[ 80 ] Anderson C, Cabana G. Does $\delta^{15} \mathrm{~N}$ in river food webs reflect the intensity and origin of $\mathrm{N}$ loads from the watershed? Science of the Total Environment, 2006, 367: 968-978.

[ 81 ] Anderson C, Cabana G. ${ }^{15} \mathrm{~N}$ in riverine food webs: effects of $\mathrm{N}$ inputs from agricultural watersheds. Canadian Journal of Fisheries and Aquatic Sciences, 2005, 62 : 333-340.

[ 82 ] Mcclelland JW, Valiela I, Michener RH. Nitrogen-stable isotope signatures in estuarine food webs: A record of increasing urbanization in coastal watersheds. Limnology and Oceanography, 1997, 42 : 930-937.

[ 83 ] Gu B, Schelske CL, Brenner M. Relationship between sediment and plankton isotope ratios $\left(\delta^{13} \mathrm{C}\right.$ and $\left.\delta^{15} \mathrm{~N}\right)$ and primary productivity in Florida lakes. Canadian Journal of Fisheries and Aquatic Sciences, 1996, 53 : 875-883.

[ 84 ] Grey J, Jones RI. Carbon stable isotopes reveal complex trophic interactions in lake plankton. Rapid Communications in Mass Spectrometry, 1999, 13: 1311-1314.

[ 85 ] Grey J, Jones RI, Sleep D. Stable isotope analysis of the origins of zooplankton carbon in lakes of differing trophic state. Oecologia, 2000, 123: 232-240.

[ 86 ] Grey J, Jones RI, Sleep D. Seasonal changes in the importance of the source of organic matter to the diet of zooplankton in Loch Ness, as indicated by stable isotope analysis. Limnology and Oceanography, 2001, 46: 505-513.

[ 87 ] Jones RI, Grey J, Sleep D et al. Stable isotope analysis of zooplankton carbon nutrition in humic lakes. Oikos, 1999, 86: $97-104$.

[ 88 ] Karlsson J, Jonsson A, Meili M et al. Control of zooplankton dependence on allochthonous organic carbon in humic and clear-water lakes in northern Sweden. Limnology and Oceanography, 2003, 48(1) : 269-276.

[ 89 ] Del Giorgio PA, France RL. Ecosystem-specific patterns in the relationship between zooplankton and POM or microplankton $\delta^{13}$ C. Limnology and Oceanography, 1996, 41 : 359-365.

[ 90 ] Ventura M, Catalan J. Incorporating life histories and diet quality in stable isotope interpretations of crustacean zooplankton. Freshwater Biology, 2008, 53: 1453-1469.

[ 91 ] Graham M. Influence of omnivory and selective feeding on $\delta^{15} \mathrm{~N}$-determined trophic position of zooplankton along a gradient of lake production. MSc. thesis. University of Regina, Regina, Sask, 1997.

[ 92 ] Leggett MF, Johannsson O, Hesslein R et al. Influence of inorganic nitrogen cycling on the $\delta^{15} \mathrm{~N}$ of Lake Ontario biota. Canadian Journal of Fisheries and Aquatic Sciences, 2000, 57 : 1489-1496.

[ 93 ] Kiriluk RM, Servos MR, Whittle DM et al. Using ratios of stable nitrogen and carbon isotopes to characterize the biomagnification of DDE, mirex, and PCB in a Lake Ontario pelagic food web. Canadian Journal of Fisheries and Aquatic Sciences, 1995,52 : 2660-2674.

[ 94 ] Bouillon S, Koedam N, Raman A et al. Primary producers sustaining macro-invertebrate communities in intertidal mangrove forests. Oecologia, 2002, 130 : 441-448.

[ 95 ] Grey J, Kelly A, Jones RI. High intraspecific variability in carbon and nitrogen stable isotope ratios of lake chironomid larvae. Limnology and Oceanography, 2004, 49: 239-244.

[ 96 ] Grey J, Kelly A, Ward S et al. Seasonal changes in the stable isotope values of lake-dwelling chironomid larvae in relation to feeding and life cycle variability. Freshwater Biology, 2004, 49: 681-689.

[ 97 ] Kelly A, Jones RI, Grey J. Stable isotope analysis provides fresh insights into dietary separation between Chironomus anthracinus and C. plumosus. Journal of the North American Benthological Society, 2004, 23 : 287-296.

[ 98 ] Bouillon S, Raman AV, Dauby P et al. Carbon and nitrogen stable isotope ratios of subtidal benthic invertebrates in an estuarine mangrove ecosystem (Andhra Pradesh, India). Estuarine Coastal and Shelf Science, 2001, 54: 901-913.

[ 99 ] Goering J, Alexander V, Haubenstock N. Seasonal variability of stable carbon and nitrogen isotope ratios of organisms in a North Pacific bay. Estuarine Coastal and Shelf Science, 1990, 30: 239-260.

[100] Ponsard S, Arditi R. What can stable isotopes $\left(\delta^{13} \mathrm{C}\right.$ and $\left.\delta^{15} \mathrm{~N}\right)$ tell about the food web of soil macro-invertebrates? Ecology, 2000, $81: 852-864$.

[101] Schmidt O, Scrimgeour CM, Curry JP. Carbon and nitrogen stable isotope ratios in body tissue and mucus of feeding and 
fasting earthworms (Lumbricus festivus). Oecologia , 1999, 118: 9-15.

[102] Lancaster J, Waldron S. Stable isotope values of lotic invertebrates: sources of variation, experimental design, and statistical interpretation. Limnology and Oceanography, 2001, 46: 723-730.

[103] Zah R, Burgherr P, Bernasconi SM et al. Stable isotope analysis of macroinvertebrates and their food sources in a glacier stream. Freshwater Biology, 2001, 46: 871-882.

[104] Mckinney RA, Nelson WG, Charpentier MA et al. Ribbed mussel nitrogen isotope signatures reflect nitrogen sources in coastal salt marshes. Ecological Applications, 2001, 11: 203-214.

[105] Fry B. Using stable isotopes to monitor watershed influences on aquatic trophodynamics. Canadian Journal of Fisheries and Aquatic Sciences, 1999, 56 : 2167-2171.

[106] Vuorio K, Tarvainen M, Sarvala J. Unionid mussels as stable isotope baseline indicators for long-lived secondary consumers in pelagic food web comparisons. Fundamental and Applied Limnology, 2007, 169 : 237-245.

[107] Gustafson L, Showers W, Kwak T et al. Temporal and spatial variability in stable isotope compositions of a freshwater mussel: implications for biomonitoring and ecological studies. Oecologia, 2007, 152 : 140-150.

[108] Lorrain A, Paulet YM, Chauvaud L et al. Differential $\delta^{13} \mathrm{C}$ and $\delta^{15} \mathrm{~N}$ signatures among scallop tissues: implications for Ecology and physiology. Journal of Experimental Marine Biology and Ecology, 2002, 275 : 47-61.

[109] Wen Z, Xie P, Xu J. Mussel isotope signature as indicator of nutrient pollution in a freshwater eutrophic lake: species, spatial, and seasonal variability. Environmental Monitoring and Assessment, 2009(10. 1007/s10661-009-0823-y).

[110] Hill JM, Mcquaid CD, Kaehler S. Biogeographic and nearshore-offshore trends in isotope ratios of intertidal mussels and their food sources around the coast of southern Africa. Marine Ecology Progress Series, 2006, 318: 63-73.

[111] Rossi F, Herman P, Middelburg JJ. Interspecific and intraspecific variation of $\delta^{13} \mathrm{C}$ and $\delta^{15} \mathrm{~N}$ in deposit-and suspensionfeeding bivalves (Macoma balthica and Cerastoderma edule) : evidence of ontogenetic changes in feeding mode of Macoma balthica. Limnology and Oceanography, 2004, 49: 408-414.

[112] Deudero S, Cabanellas M, Blanco A et al. Stable isotope fractionation in the digestive gland, muscle and gills tissues of the marine mussel Mytilus galloprovincialis. Journal of Experimental Marine Biology and Ecology, 2009, 368 : 181-188.

[113 ] Mckinney RA, Lake JL, Allen M et al. Spatial variability in mussels used to assess base level nitrogen isotope ratio in freshwater ecosystems. Hydrobiologia, 1999, 412: 17-24.

[114] Fukumori K, Oi M, Doi H et al. Bivalve tissue as a carbon and nitrogen isotope baseline indicator in coastal ecosystems. Estuarine, Coastal and Shelf Science, 2008, 79: 45-50.

[115] Raikow DF, Hamilton SK. Bivalve diets in a midwestern US stream: A stable isotope enrichment study. Limnology and Oceanography, 2001, 46: 514-522.

[116] Hawkins AJS. Relationships between the synthesis and breakdown of protein, dietary absorption and turnovers of nitrogen and carbon in the blue mussel, Mytilus edulis L. Oecologia, 1985, 66: 42-49.

[117] Kurata K, Minami H, Kikuchi E. Stable isotope analysis of food sources for salt marsh snails. Marine Ecology Progress Series, 2001, 223: 167-177.

[118] Vander Zanden MJ, Vadeboncoeur Y. Fishes as integrators of benthic and pelagic food webs in lakes. Ecology, 2002, 83 : 2152-2161.

[119] Burns TP. Lindeman's contradiction and the trophic structure of ecosystems. Ecology, 1989, 70: 1355-1362.

[120] Ernest SK, Enquist BJ, Brown JH et al. Thermodynamic and metabolic effects on the scaling of production and population energy use. Ecology Letters, 2003, 6: 990-995.

[121] Vaner Zanden MJ, Rasmussen JB. Variation in $\delta^{13} \mathrm{C}$ and $\delta^{15} \mathrm{~N}$ trophic fractionation: implications for aquatic food web studies. Limnology and Oceanography, 2001, 46: 2061-2066.

[122] Xu J, Zhang M, Xie P. Trophic relationship between the parasitic isopod Ichthyoxenus japonensis and the fish Carassius auratus auratus as revealed by stable isotopes. Journal of Freshwater Ecology, 2007, 22 : 333-338.

[123] Harvey CJ, Hanson PC, Essington TE et al. Using bioenergetics models to predict stable isotope ratios in fishes. Canadian Journal of Fisheries and Aquatic Sciences, 2002, 59: 115-124.

[124] Hesslein RH, Hallard KA, Ramlal P. Replacement of sulfur, carbon, and nitrogen in tissue of growing broad whitefish (Coregonus nasus) in response to a change in diet traced by $\delta^{34} \mathrm{~S}, \delta^{13} \mathrm{C}$ and $\delta^{15} \mathrm{~N}$. Canadian Journal of Fisheries and Aquatic Sciences, 1993, 50: 2071-2076. 